\title{
ILLUMINATION INVARIANT ACTIVE CONTOUR-BASED SEGMENTATION USING COMPLEX-VALUED WAVELETS
}

\author{
Alexander Wong \\ Department of Systems Design Engineering \\ University of Waterloo \\ Waterloo, Ontario, Canada
}

\begin{abstract}
This paper introduces a novel approach to the problem of active contour-based segmentation through the use of complexvalued wavelets. In traditional active contour-based segmentation techniques based on level set methods, the energy functionals are defined based on intensity gradients. This makes them highly sensitive to situations where the underlying image content is characterized by image non-homogeneities due to illumination and contrast conditions. In the proposed approach, the energy functionals used to evolve a level set function are based on the moments of phase coherence of complex-valued wavelet components. This formulation is highly invariant to non-homogeneities caused by illumination and contrast variations. Experimental results demonstrate that the proposed approach can be used to improve existing active contour-based segmentation methods under situations characterized by image non-homogeneities.
\end{abstract}

Index Terms - active contour, segmentation, complexvalued wavelets

An increasingly popular area of research in the field of image processing is image segmentation, where the underlying goal is to partition image content into multiple regions in a meaningful manner. Some recent work in image segmentation have focused on active contours based on level set methods [1, 2, 3], where active contours are represented as the zero level set of a level set function, which is then evolved based on a partial differential equation towards the minimum cost potential. In existing image segmentation methods based on level set active contours, the energy functionals that act as a basis of cost minimization for evolving the active contours are based on the intensity gradients of the underlying image content. The main drawback to using intensity gradients as the basis for the energy functional is that it makes evolution process highly sensitive to image non-homogeneities caused by illumination and contrast conditions. As such, minimizing the cost potential based on intensity gradients will often result in poor image segmentation in situations where the image is acquired under non-uniform illumination conditions. As such, an approach for constructing energy functionals that are highly robust by illumination and contrast conditions is desired.

The main contribution of this paper is a novel approach to the problem of level set active contour-based image segmentation through the use of energy functionals defined based on the phase coherence of complex-valued wavelets. The proposed approach can be integrated into existing level set active contour-based image segmentation techniques to provide improved segmentation performance in situations characterized by illumination non-homogeneities. The proposed approach extends upon the variational formulation for level set active contours proposed in [3] in such a way that the resulting image segmentation process is largely invariant to illumination conditions. This paper is organized as follows. The proposed approach is described in 1. Experimental results are presented and discussed in Section 2. Finally, conclusions are drawn in Section 3.

\section{PROPOSED APPROACH}

The proposed approach to illumination invariant active contourbased image segmentation utilizes the moments of phase coherence of complex-valued wavelet components to define energy functionals. Therefore, the first step in the proposed approach is to compute the moments of phase coherence from a given image. Suppose we are given an image $I$ that we wish to segment. The amplitude $A$ and phase $\phi$ at a given point can be obtained using complex wavelets such as Gabor wavelets and dual-tree complex wavelets [4]. For example, using logarithmic Gabor wavelets, the local amplitude and phase for a given pixel at $\underline{x}$ and wavelet scale $n$ can be computed using the following equations:

$$
\begin{gathered}
A_{n}(\underline{x})=\sqrt{\left(I(\underline{x}) * F_{n}^{e}\right)^{2}+\left(I(\underline{x}) * F_{n}^{o}\right)^{2}} \\
\phi_{n}(\underline{x})=\tan ^{-1}\left(\frac{\left(I(\underline{x}) * F_{n}^{e}\right)}{\left(I(\underline{x}) * F_{n}^{o}\right)}\right)
\end{gathered}
$$

where $F_{n}^{e}$ and $F_{n}^{o}$ are the even-symmetric and odd-symmetric wavelets at scale $n$ respectively. Based on this local frequency information, the phase coherence of the complex-valued 
wavelet components can be determined. The phase coherence of frequency and wavelet components contains information that signifies the structural variations within an image and has been used in different applications where these variations are important in the image analysis process $[5,6,7]$. Unlike intensity gradients, which relies heavily on amplitudinal information of an image, the phase coherence relies mainly on the phase information of an image and is therefore highly invariant to image non-homogeneities. Using the formulation described in [6], the phase coherence of a pixel at $\underline{x}$ and orientation $\theta$ can be estimated as follows:

$$
P(\underline{x}, \theta)=\frac{\sum_{n} W(\underline{x}, \theta)\left\lfloor A_{n}(\underline{x}, \theta) \Delta \Phi(\underline{x}, \theta)-T\right\rfloor}{\sum_{n} A_{n}(\underline{x}, \theta)+\varepsilon}
$$

$\Delta \Phi(\underline{x}, \theta)=\cos \left(\phi_{n}(\underline{x}, \theta)-\bar{\phi}(\underline{x}, \theta)\right)-\left|\sin \left(\phi_{n}(\underline{x}, \theta)-\bar{\phi}(\underline{x}, \theta)\right)\right|$

where $W$ represents the frequency spread weighting factor (coherence over wider frequency spreads outweighs coherence over narrow frequency spreads), $\bar{\phi}$ represents the weighted mean phase, $T$ represents the noise threshold and $\varepsilon$ is a small constant used to avoid division by zero. $\Delta \Phi$ goes to zero when the complex-valued wavelet components are minimally in phase, and goes to approximately one when they are maximally in phase. Most of the parameters used to compute phase coherence are the same as that used in [6], except $n=3$.

Once the phase coherence of the wavelet components have been determined, the maximum moments of phase coherence $\varpi$ can be computed as follows:

$$
\frac{1}{2} \sum_{\theta} P(\underline{x}, \theta)^{2}+\frac{1}{2} \sqrt{\begin{array}{l}
4\left(\sum_{\theta}(P(\underline{x}, \theta) \sin (\theta))(P(\underline{x}, \theta) \cos (\theta))\right)^{2}+ \\
\left(\sum_{\theta}\left[(P(\underline{x}, \theta) \cos (\theta))^{2}-(P(\underline{x}, \theta) \sin (\theta))^{2}\right]\right)^{2}
\end{array}}
$$

where $P(\underline{x}, \theta)$ is the phase coherence at $\underline{x}$ and orientation $\theta$. High values of $\varpi$ corresponds with high structural variations while low values of $\varpi$ corresponds with low structural variations. In this context, the maximum moments of phase coherence can be conceptually viewed as a form of image gradient and can therefore be integrated into the energy functional used to minimize cost potential for the level set function. Using the level-set active contour-based image segmentation method proposed by Li et al. [3] as the basis of the proposed approach, the external energy functional $E$ can be defined as follows:

$$
E(\phi)=\lambda \int \frac{1}{1+\varpi^{2}} \delta(\phi)|\nabla \phi| d \underline{x}+\nu \int \frac{1}{1+\varpi^{2}} H(-\phi) d \underline{x}
$$

where $\lambda$ and $\nu$ are constants $\left(\lambda=5\right.$ and $\nu=\frac{3}{2}$ during testing), $\delta$ is the univariate Dirac function, and $H$ is the Heaviside function. This can then be used to define the total energy functional as proposed in [3]:

$$
C(\phi)=\mu \int \frac{1}{2}(|\nabla \phi|-1)^{2} d \underline{x}+E(\phi)
$$

where $\mu$ is a constant $\left(\mu=\frac{1}{25}\right)$.

\section{EXPERIMENTAL RESULTS}

A group of test images were used to test the effectiveness of the proposed method. As stated earlier, the proposed approach can be used to improve existing level set active contour-based techniques. For testing purposes, the proposed approach was integrated into the level-set active contourbased image segmentation method proposed by Li et al. [3]. To evaluate the performance of the proposed approach, both the original approach used in Li's method for energy functionals and that proposed in this paper are compared over 300 iterations. Example segmented images are shown in Figure 1, Figure 2 and Figure 3. It can be seen that the proposed method provides improved segmentation in both cases, particularly in areas with high image non-homogeneities. These results show that the proposed approach can be used to improve existing level set active contour-based techniques in situations characterized by image non-homogeneities.
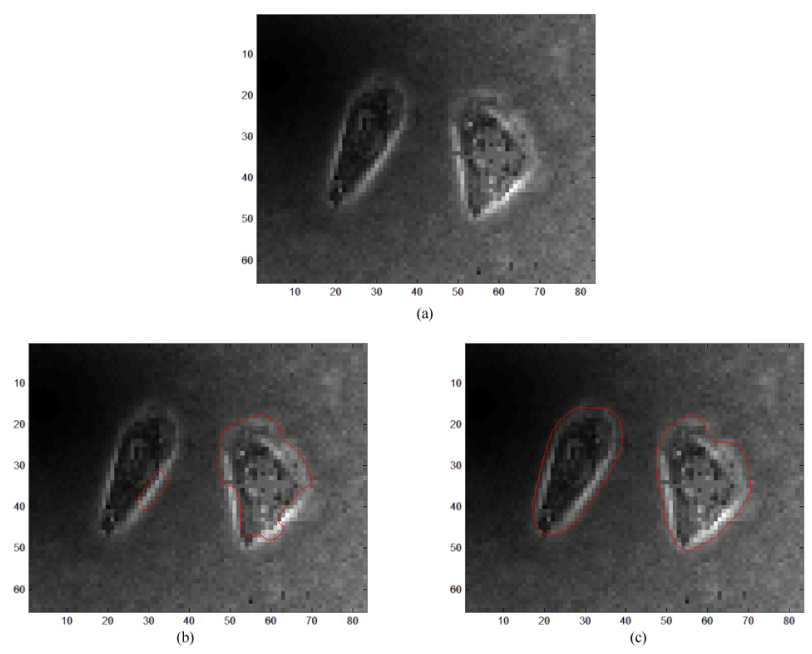

Fig. 1. TEST1: Top: Original image, Bottom-right: Segmentation using Li's method, Bottom-right: Segmentation using proposed approach

\section{CONCLUSIONS}

This paper introduced a novel approach to illumination invariant level set active contour-based image segmentation using complex-valued wavelets. By utilizing moments of phase coherence of complex-valued wavelet components in the energy functionals, it was demonstrated that improved segmentation quality can be achieved over the approach used in current level set active contour-based segmentation techniques. 

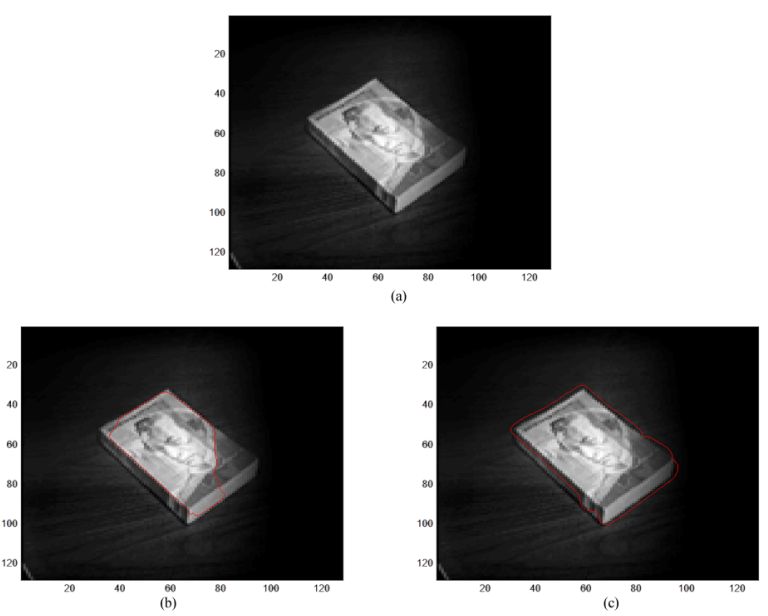

Fig. 2. TEST2: Top: Original image, Bottom-right: Segmentation using Li's method, Bottom-right: Segmentation using proposed approach
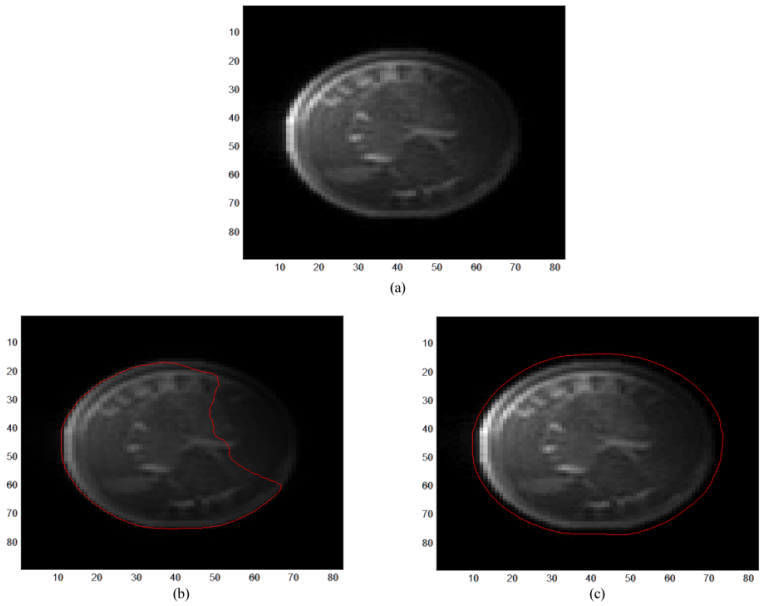

Fig. 3. TEST3: Top: Original image, Bottom-right: Segmentation using Li's method, Bottom-right: Segmentation using proposed approach
Finally, the proposed approach can be integrated into existing segmentation techniques to improve segmentation quality. Future work includes investigating methods for improving segmentation performance of the proposed approach under very high levels of noise, where the moments of phase coherence becomes highly degraded.

\section{Acknowledgment}

The authors would like to thank the Natural Sciences and Engineering Research Council (NSERC) of Canada for funding this project. The authors would also like to thank the Vision and Image Processing Group at the University of Waterloo.

\section{REFERENCES}

[1] V. Caselles, F. Catte, T. Coll, and F. Dibos, A geometric model for active contours in image processing, $\mathrm{Nu}$ merische Mathematik, vol. 66, pp. 1-31, 1993.

[2] R. Malladi, J. Sethian, and B. Vemuri, Shape modeling with front propagation: a level set approach, IEEE Transactions on Pattern Analysis and Machine Intelligence, vol. 17, pp. 158-175, 1995.

[3] C. Li, C. Xu, C, Gui, and M. Fox, "Level set evolution without re-initialization: a new variational formulation," Proceedings of the IEEE Computer Society Conference on Computer Vision and Pattern Recognition, vol. 1, pp. 430-436, 2005.

[4] I. Selesnick, W. Ivan , R. Baraniuk, and N. Kingsbury, "The Dual-Tree Complex Wavelet Transform", IEEE Signal Processing Magazine, vol. 22, no. 6, pp. 123-151, 2005.

[5] M. Morrone and D. Burr, "Feature detection in human vision: A phase-dependent energy model," Proc. Royal Society of London B, vol. 235, pp. 221-245, 1988.

[6] P. Kovesi, "Phase congruency detects corners and edges," Proc. Australian Pattern Recognition Society Conference, pp. 309-318, 2003.

[7] Z. Wang and E. Simoncelli, "Local phase coherence and the Perception of Blur,' Advances in Neural Information Processing Systems, vol. 16, May 2004. 